\title{
Preliminary Design of a Mach 6 Configuration using MDO
}

\author{
Robert Dittrich and José M.A. Longo \\ German Aerospace Center (DLR) - Institute of Aerodynamics and Flow Technology \\ Lilienthalplatz 7, 38108 Braunschweig, Germany \\ robert.dittrich@dlr.de
}

\begin{abstract}
Summary
The paper presents a multidisciplinary optimization (MDO) process for the analysis of configurations with engines strongly integrated into the airframe. The coupled treatment of several physical disciplines like aerodynamics, flight mechanics, propulsion and structure is discussed taking into account the major steps of a flight envelope of a hypersonic transport aircraft like transonic acceleration, hypersonic cruise and subsonic landing. The technique is successfully applied optimizing a Mach 6 transport aircraft considering a single as well as multiple flight conditions. In both cases the optimization technique allows to improve the cruise range of the vehicle.
\end{abstract}

\section{Introduction}

In the frame of the EU ATLLAS Project (Aerodynamic and Thermal Load Interactions with Lightweight Advanced Materials for High Speed Flight) the appropriate requirements for the airframe and propulsion materials of future high speed transport airplanes are derived from two configurations, a Mach 3 supersonic and a Mach 6 hypersonic transport. For the Mach 6 configuration is not the aim of the ATLLAS project to design a specific vehicle but to explore todays state of the art technology limits to realize such kind of concept.

Hypersonic atmospheric vehicles are propelled either by ram- or scramjet type of engines, i.e. a kind of propulsion system which requires a successful integration into the airframe in order to avoid major propulsion-efficiency losses. Such propulsionintegration has received considerable attention during the eighties. While it was recognized the need of use massively CFD in order to achieve a successful design, CFD was at that time almost in its infancy. However, today a multidisciplinary optimization technique could provide the right platform for the realization of such kind of vehicle as is the aim to demonstrate in the present work.

\section{Reference Design Configuration}

In order to carry out a MDO process, the definition of the vehicle to be optimized shall be done in a way that sufficient information about engine performance and 
structure layout are available. Since EU projects are strongly limited in time and resources to perform a completely new design, it was here decided to select from open literature of past studies a baseline configuration. Only configurations which match the reference ATLLAS mission, i.e. transportation of 200 passengers over a distance of $7000 \mathrm{~km}$ at a cruise Mach number 6 and an altitude about 30km, while providing the major amount of technical data have been considered. In view these criteria, the HYCAT-1A [1][2] has been selected as baseline for a future MDO process. The availability of a huge database including wind tunnel tests and the promising compromise between hypersonic and subsonic performance as well as good trim capabilities, both major requirements for a realistic hypersonic aircrafts, favor the HYCAT-1A. This vehicle has a classical horizontal tail, characteristic sharp forebody leading edge which merges to the wing leading edge and it is driven by a combined turbojet-ramjet engine based on hydrogen fuel. The fuselage is 105 meter long with a spanwidth of 28 meters.

Being rigorous, the baseline vehicle for the MDO process, namely the ATLLAS Mach 6 reference configuration, is not equal to the HYCAT-1A but similar. Also corresponding mass budget estimation, turbo-ramjet performances, mission profile, aerodynamic database, structure analysis and trim capabilities have been specifically generated for the baseline configuration while those of the HYCAT-1A are used for crosscheck purpose. In such a way, the most critical issues of the configuration can be indicated and hence major objectives and important system requirements and constraints for the MDO can be formulated. In particular here the major issues that have to be considered during the MDO process are (1) the mandatory integration of the engine due to the lift increase, (2) the identification of the end of cruise phase with worst trim conditions and (3) the low frequency lateral and vertical bending of the configuration due to the large dimensions.

\section{MDO Tool}

The MDO tool consists of several modules for different subtasks which are added to a function chain where at the end a defined objective function is updated. The workflow for a 3-point MDO process is demonstrated in Figure 1 and is generally defined by parameterized geometry generation, mass modelling for component masses and centre of gravity computation, CFD grid generation, numerical aerodynamic flow solving, thrust and trim capability determination, FEM grid generation and dynamic structure analysis, constraints check and objective function update. Most of the modules are also depending on the flight regime e.g. transonic or cruise conditions. Concerning the MDO this has mainly a big impact on the propulsion system integration. Hence geometrical and physical differences of the engine in different mission points are considered.

The MDO tool includes both, hypersonic critical issues as well as general MDO relevant aspects. The propulsion system is integrated in the MDO in a form that intake and nozzle flow is directly computed in the CFD and the combustion chamber is covered as a black box with given properties so that the gross thrust can be determined. 
To speed up the MDO process special methods are developed like a modular mesh generation procedure which strongly reduces meshing time. The MDO tool is built up using some commercial software but also using own developed source codes. All modules are embedded in a new and fully automated PYTHON environment taking over running and monitoring of modules, data exchange and conversion, machine communication and database update. The modular concept of the MDO process allows simple removing, adding and modifying of modules.

The MDO tool is linked to the commercial software SYNAPS POINTER PRO [7] which offers several types of optimizers. In the presented MDO the Subplex optimizer, a function ranking method, is favoured. Below the basic modules of the MDO tool are shortly presented.

\subsection{Geometry Generation}

The geometry generation is one of the major modules of the MDO tool because most of the engaged modules are depending on the geometry. For the geometry generation an own tool is developed based on NURBS curves [3] by defining control points. A certain number of NURBS curves are arranged in 3D-space resulting in a surface. The geometry is divided in several surfaces and changing NURBS attributes offers different kinds of surface interfaces from complete smooth to kinked ones. The geometry description is completely parameterized, so that the airframe is controlled by about 100 parameters and the engine by 40 parameters. Figure 2 shows the generated geometry of the reference design. The tool allows global and local geometry changes modifying NURBS control points and guarantees water closed geometry. Additionally inner surfaces for tanks and passenger cabin are created. Furthermore the geometry tool can be used directly for node creation needed for structure models.

\subsection{FEM Calculation}

An initial FEM model provided by ATLLAS partner FOI is adapted to the MDO process including automated mesh generation connected to the geometry procedure. The model consists of 4 nodes shell elements for cover plates, bar elements simulating frame stations, spars and stringers and rigid body elements for component connections. For FEM computations the numerical structure solver NASTRAN is used with concentration on dynamic eigenvalue analysis [6] to consider critical bending modes of the configuration as demonstrated in Figure 3.

\subsection{Mass Estimation}

For the mass estimation the initial mass budget is splitted into constant and geometry depending masses in form of mass distributions. Applying a new configuration geometry now surface areas and geometrical centre of gravity of these surfaces are determined. Every surface is then loaded with a constant mass and a mass distribution given by the splitted mass budget. In combination with the new calculated tank volumes component masses, fuel masses and centre of gravity depending on fuel charging can be updated, see Figure 4. 


\subsection{CFD Grid Generation}

Allowing large geometry changes during the MDO re-meshing of the CFD grid within every optimization loop is needed. Therefore the commercial unstructured grid generator CENTAUR [5] is used. For higher accuracy grids with about 1.8 million nodes are used where almost half of the nodes reside inside the engine zone. Suitable source placement guarantees constant mesh refinement for certain local geometry parts like wing leading edges. For an optimization procedure covering 3 flight conditions it is necessary to use 3 different meshes due to (1) the different engine modes, (2) the different deflections of the horizontal stabilizer and (3) the CFD requirements in far field for each flight conditions. It turns out grid generation is one of the main driver for the overall loop time. So a special modular grid generation procedure is developed by splitting the 3D-field around the configuration into several zones which can be re-meshed independently, see Figure 5(a). Only zones where the geometry changes have to be re-meshed and then grids for different flight conditions are created by grid uniting of main, engine and horizontal stabilizer zone. Hence the overall meshing time during one loop is strongly reduced.

\subsection{CFD TAU Calculation}

The CFD calculations are performed using the DLR TAU code [8], a Reynoldsaveraged Navier-Stokes flow solver applicable for subsonic as well as hypersonic cases. For reducing flow solver time TAU is running in Euler mode in addition with large parallel computing. The drag due to skin friction is taken into account after CFD calculation by a turbulent flat plate model. Fast convergence is reached using three level multigrid, 2nd order AUSMDV upwind scheme for flux discretization and three step Runge-Kutta method for relaxation solving. The targeted lift is provided by the mass estimation hence the resulting angle of attack and flow field is numerically computed. Figure 5(b) shows a Mach number plot for cruise conditions including a zoom to the engine where the outer engine walls are set to invisible to show intake compression and nozzle expansion.

\subsection{Force and Trim Calculation}

The force balance is calculated from the CFD results plus a force model for the black box combustion chamber presented in Figure 6(a) including the gross thrust and small intake corrections. Forces for intake and nozzle are already included in the CFD calculation. Thus the main force coefficients for lift, drag, thrust and pitch moment are computed. To determine the trim capability, the location of the pressure point is computed based on the above information and compared with the centre of gravity. Here plays an important role the effectiveness of the horizontal-stabilizer deflection, which as Figure 6(b) brings out decreases as the Mach number increases. On the other side the specific fuel consumption is calculated from the net thrust given by intake, combustion chamber and nozzle force and fuel mass flow for the current engine mode. 


\subsection{Objective Function and Constraints Handling}

As objective function for the MDO process it is chosen the range due to linkage of aerodynamic and engine performance as well as fuel and operating empty mass.

For an optimization considering only one flight condition, the Breguet range is used. For a multiple flight-condition optimization, a new expression for the range is evaluated by integrating the basic range equation for un-accelerated horizontal flight.

The configuration constraints which can not be found in the range equation are added to the objective function in form of a penalty function which gives the final objective function. Hence the constrained optimization problem is changed to an unconstrained optimization problem. As disadvantage of this method a noisy objective function characteristic is expected. Main constraints are: (i) the intake air mass flow for begin of cruise, (ii) the distance between centre of gravity and pressure point for all calculated mission points, (iii) the gross lift off weight and (iv) the resulting force in flight direction for all cruise points.

\subsection{Optimizer}

As mentioned in the beginning the Subplex optimizer is applied for the MDO process. The Subplex optimizer is based on the Nelder-Mead simplex (NMS) method which is often recommended as best optimizer for noisy function due to a function value ranking system which is not depending on absolute objective function values. Furthermore no parameter sensitivity study is necessary, but NMS is limited to low dimensional problems $(n<6)$. The Subplex optimizer now makes the NMS feasible for high dimensional problems by determining subspaces of the parameter space where the NMS can be applied, a so called subplex cycle is evaluated. Convergence can be observed after three till five subplex cycles [4].

\section{MDO Applications}

The structural module is not included in MDO processes below. A first MDO considering only one flight condition, begin of cruise, has been performed to validate the functionality of the MDO tool. In every loop the targeted lift is determined by the configuration gross lift off weight calculated by the mass module minus a constant fuel mass for acceleration and climb. The targeted lift is then given as input for the CFD calculations. Overall 13 geometrical design parameters, 4 for wing, 4 for horizontal stabilizer and 5 for fuselage have been chosen. The result of the optimization for a single flight condition is shown in figure 7(a). There the initial configuration is compared against the optimized one. The cruise range is increased by 10 percent due to increase of L/D and tank volume without losing aerodynamic performance. Then an optimization considering 3 flight conditions has been realized as described in Figure 1 by adding the transonic acceleration phase $(M=1.3)$ and the end of cruise phase (this last due to the critical trim condition mentioned above). The configuration mass at begin of cruise is now depending on the transonic performance 
which determines fuel consumption during acceleration and climb. The number of design parameters has increased up to 22. Assuming lift is proportional to mass, constant cruise velocity and flight height the basic range equation is integrated in a form that the aerodynamic performance at end of cruise is included in the cruise range calculation. Figure 7(b) presents the current results for the multiple flight conditions optimization. The objective function $(\mathrm{OF})$ is defined by the range to initial range ratio $\frac{R}{R_{\text {init }}}$ divided by the penalty function $p$. The Figure shows the evolution of the configuration along the MDO process. The optimization process leads out a system with an increased objective function by 9 percent.

\section{Conclusions}

A new MDO tool with application to a Mach 6 hypersonic configuration has been presented. The initial design, major requirements and important constraint formulations for the MDO process are discussed followed by the description of the automated modules for the different subtasks. Three of four targeted disciplines are considered while a structural modeling concerning dynamic eigenvalue analyses is prepared for its implementation in the MDO tool. The modular build-up of the MDO tool allows modifying several modules for future improvements. The functionality of the MDO tool is demonstrated for a single as well as for a multiple flight conditions resulting in all the cases in an increase of the cruise range.

\section{Acknowledgment}

This work is being performed within the 'Aerodynamic and Thermal Load Interactions with Lightweight Advanced Materials for High Speed Flight' project ATLLAS, coordinated by ESA-ESTEC and supported by the EU within the 7th Framework Program, Aeronautic and Space, Contract no.: AST5-CT-2006-030729.

\section{References}

[1] Brewer, G.D; R.E. Morris, R.E.: Hypersonic Cruise Aircraft Propulsion Integration Study Volume I/II, NASA Contractor Report, CR-158926-1, 1979

[2] Ellison, J.C.: Investigation of the Aerodynamic Characteristics of a Hypersonic Transport Model at Mach Numbers to 6, NASA Technical Note D-6191, 1971

[3] Piegl, L.: The NURBS Book, 2nd Edition, Springer, 1997

[4] Rowan, T.: Functional Stability Analysis of Numerical Algorithms, Thesis, Department of Computer Sciences, University of Texas at Austin, USA, 1990

[5] CENTAUR Version 7.5 B1, CentaurSoft, 2007, www.centaursoft.com 
[6] MSC NASTRAN 2007 rl Quick Reference Guide, MSC.Software Corporation, Santa Ana, USA, 2007

[7] SynapsPointer Pro 2, Synaps Ingenieur-Gesellschaft mbH, Bremen, Germany, 2003

[8] TAU Technical Report, DLR, Institute of Aerodynamics and Flow Technology, Braunschweig, Germany, 2007

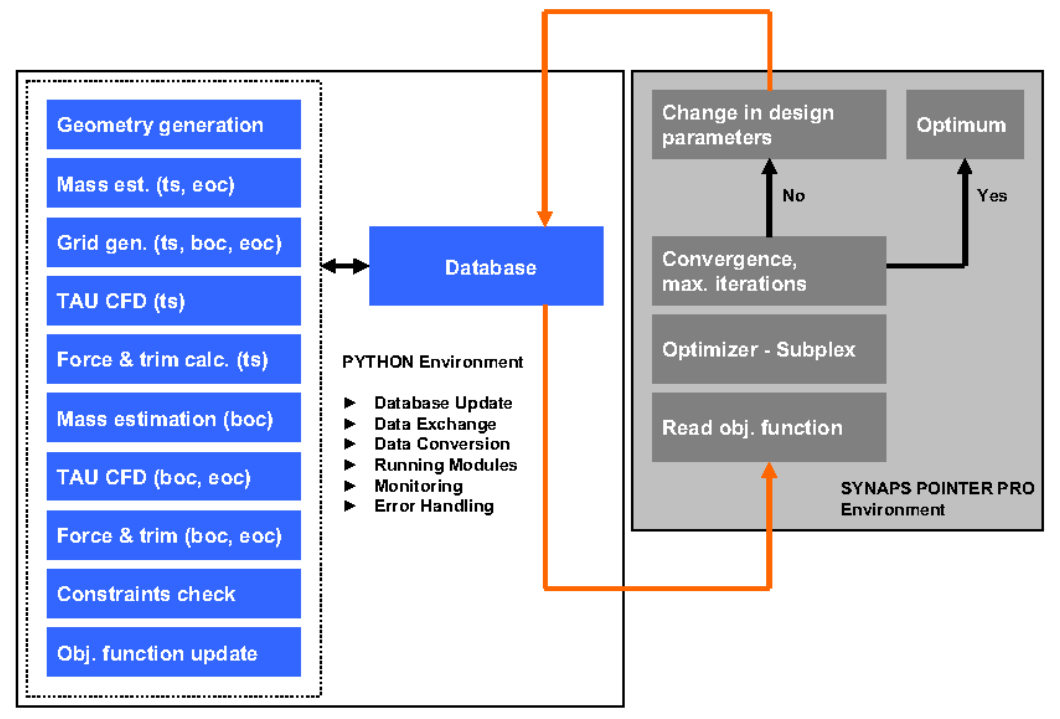

Figure 1: Flow chart for 3-point MDO process

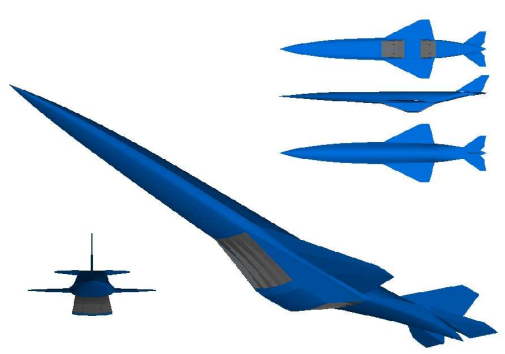

Figure 2: Reference design geometry

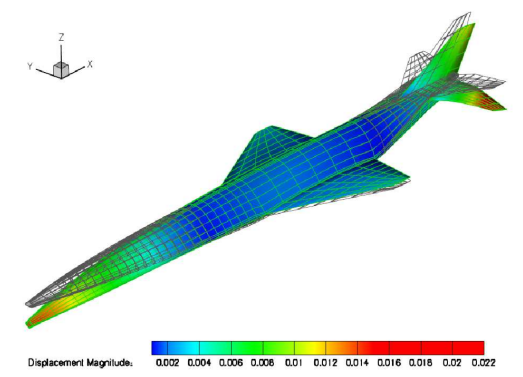

Figure 3: FEM: vertical bending mode 


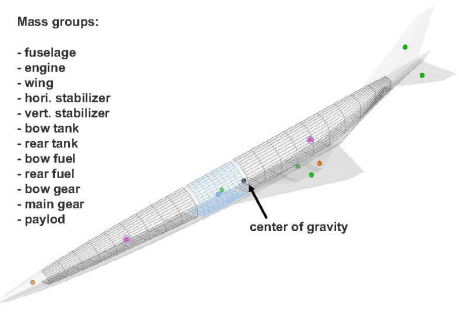

(a) Mass components

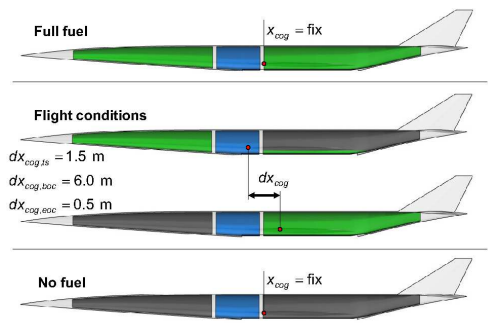

(b) COG influence due to fuel charging

Figure 4: Mass and centre of gravity modelling

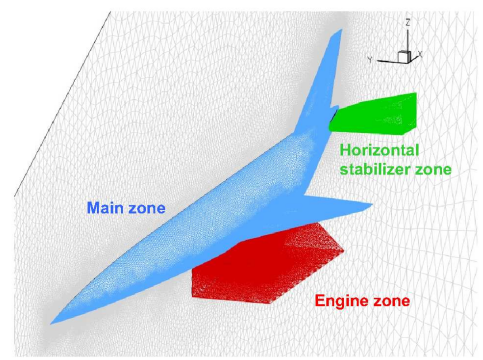

(a) Grid zones of modular CFD grid

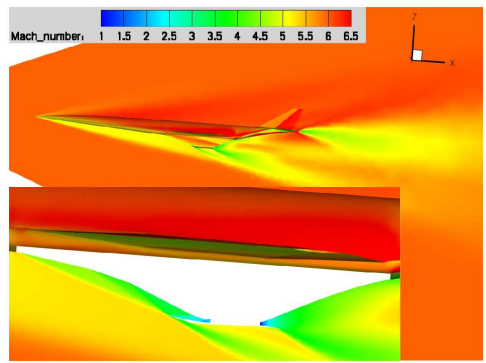

(b) Mach number plot for $\mathrm{M}=6.0$ Figure 5: TAU CFD calculation

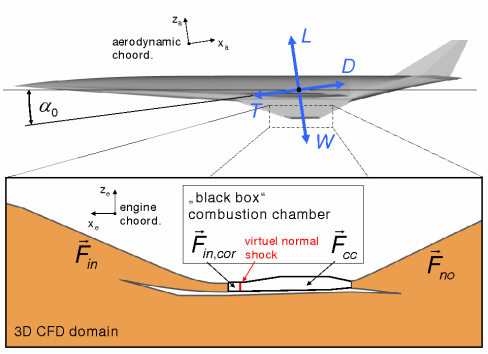

(a) Force definitions

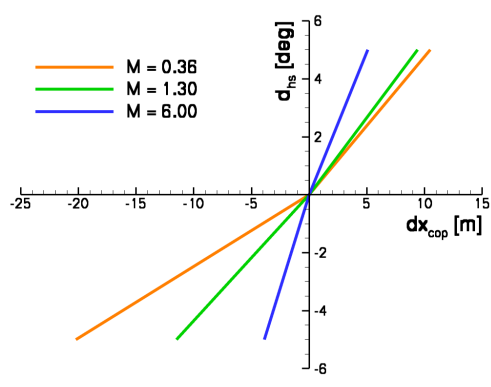

(b) Horizontal stabilizer performance Figure 6: System forces and horizontal stabilizer performance
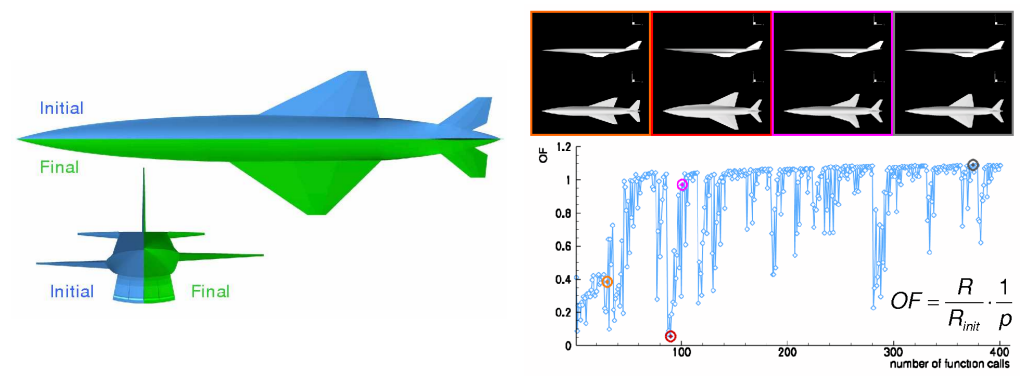

(a) 1-point MDO result (b) Characteristics of 3-point MDO

Figure 7: MDO applications 